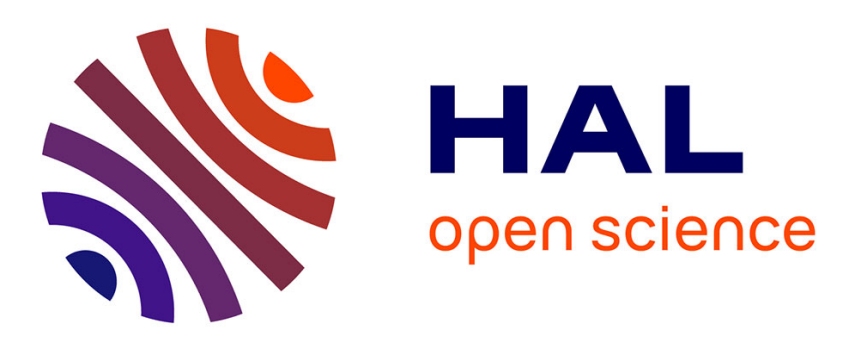

\title{
Control of Intermolecular Photoinduced Electron Transfer in Deoxyadenosine-Based Fluorescent Probes
}

Hoang-ngoan Le, Johanna Brazard, Guillaume Barnoin, Steve Vincent, Benoît y Michel, Jérémie Leonard, Alain Burger

\section{- To cite this version:}

Hoang-ngoan Le, Johanna Brazard, Guillaume Barnoin, Steve Vincent, Benoît y Michel, et al.. Control of Intermolecular Photoinduced Electron Transfer in Deoxyadenosine-Based Fluorescent Probes. Chemistry - A European Journal, 2021, 27 (4), pp.1364-1373. 10.1002/chem.202003456 . hal03357459

\author{
HAL Id: hal-03357459 \\ https://hal.science/hal-03357459
}

Submitted on 19 Nov 2021

HAL is a multi-disciplinary open access archive for the deposit and dissemination of scientific research documents, whether they are published or not. The documents may come from teaching and research institutions in France or abroad, or from public or private research centers.
L'archive ouverte pluridisciplinaire $\mathbf{H A L}$, est destinée au dépôt et à la diffusion de documents scientifiques de niveau recherche, publiés ou non, émanant des établissements d'enseignement et de recherche français ou étrangers, des laboratoires publics ou privés. 


\title{
Control of Intermolecular Photoinduced Electron Transfer in Deoxyadenosine-Based Fluorescent Probes
}

\author{
Hoang-Ngoan Le, ${ }^{\mathrm{a}, \dagger}$ Johanna Brazard,,${ }^{\mathrm{b},}$ Guillaume Barnoin, ${ }^{\mathrm{a}}$ Steve Vincent, ${ }^{\mathrm{a}}$ Benoît Y. Michel, ${ }^{\mathrm{a}}$ \\ Jérémie Leonard, ${ }^{\mathrm{b}}$ Alain Burger ${ }^{\mathrm{a}}$ \\ ${ }^{a}$ Université Côte d'Azur, CNRS, Institut de Chimie de Nice, UMR 7272 - Parc Valrose, 06108 Nice cedex 2, France \\ ${ }^{b}$ Université de Strasbourg, CNRS, Institut de Physique et Chimie des Matériaux de Strasbourg and Labex NIE, UMR 7504, F-67200 Strasbourg, \\ France \\ † Main contributor to these research works \\ * Present address: Université de Genève, Département de Chimie Physique, CH-1211 Genève
}

\section{Graphical abstract:}

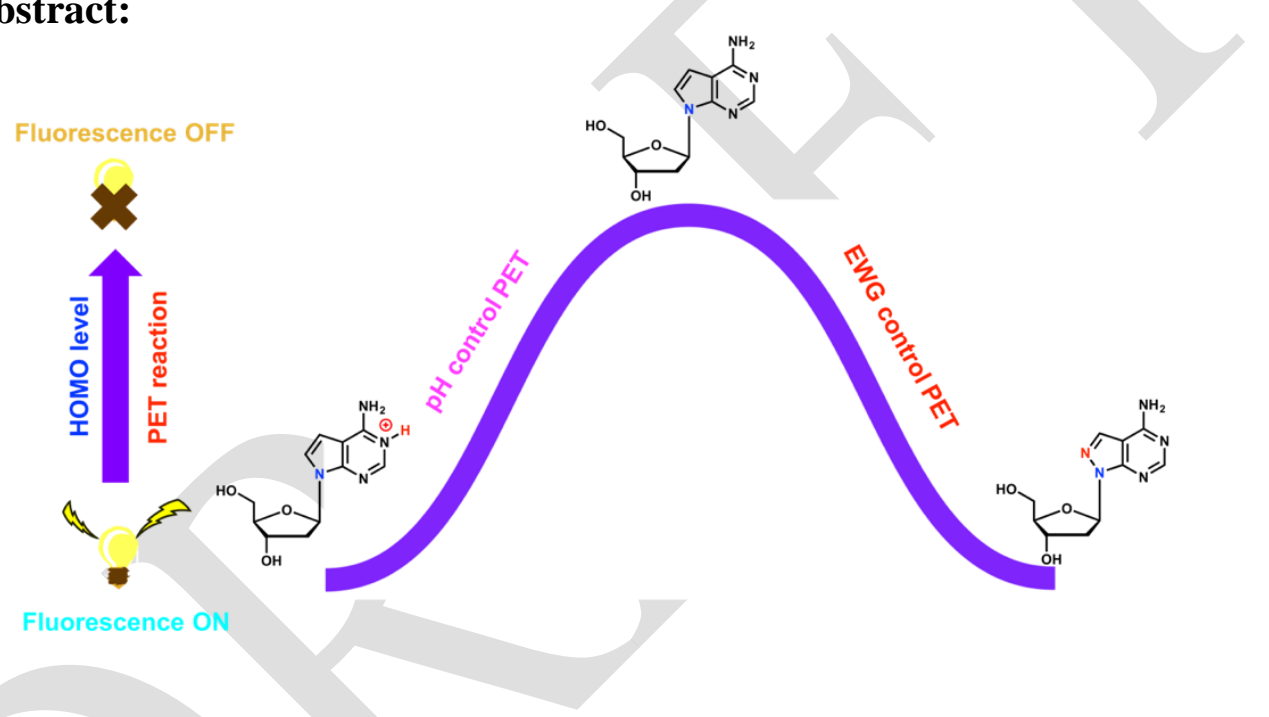

\section{Abstract}

In this paper, we report on the Photoinduced Electron Transfer (PET) reaction between a donor (adenine analog) and an acceptor (3-methoxychromone derivative) in the context of designing efficient fluorescent probes as DNA sensors. Firstly, Gibbs energy was investigated in disconnected donor-acceptor systems by Rehm-Weller equation. The oxidation potential of the adenine derivative was responsible for exergonicity of the PET reaction in separated combinations. Then, the PET reaction in donor- $\pi$-acceptor conjugates was investigated using steady-state fluorescence spectroscopy, acid-mediated PET inhibition and transient absorption techniques. In conjugated systems, PET is a favorable pathway of fluorescent quenching when an electron-rich adenine analog (d7A) was connected to the fluorophore (3MC). We found that formation of ground-state complexes even at $\mathrm{nM}$ concentration range dominated the dye photophysics and generated poorly emissive species likely through intermolecular PET from d7A to 3MC. On the other hand, solution acidification disrupts complexation and turns on dye emission. Bridging an electron-poor adenine analog with high oxidation potential $(\mathbf{8 d 7 A})$ to $3 \mathbf{M C}$ presenting low reduction potential is another alternative to prevent complex formation and produce highly emissive monomer conjugates. 
Keywords: Ground-state complex, photoinduced electron transfer, push-pull dye, deoxyadenosine, turn-on

\section{Introduction}

Redesigning the structure of nucleosides to generate environment-sensitive fluorescent analogs and incorporating them into nucleic acids (NA) is a leading approach for the development of NArelated advanced sensors for sequencing and probing NA conformations, dynamics and interactions ${ }^{[1-3]}$. A common strategy is grafting a chromophore to the nucleobase preferentially on a position not affecting Watson-Crick base pairing. In term of biocompatibility, extension at positions 5 and 7 of pyrimidine and purine rings respectively, is the most favorable ${ }^{[1,4,5]}$. Connecting a fluorophore is attractive as it opens the route to the development of emissive probes based on sensing characteristics of the original dye ${ }^{[6]}$. Extension of nucleobases electronic $\pi$ scaffolds to construct push-pull systems is particularly interesting since it could generate probes with unique photophysical properties including sensitivity to polarity and red-shifted absorption and emission ${ }^{[7]}$. The responsiveness of the fluorescence reporter can be greatly increased if the emissive signal is based on the change between two spectrally resolved forms ${ }^{[8]}$. In order to achieve this, we have selected fluorophores of the 3-HydroxyChromone (3HC) family that can exist in two excited-state forms - the normal and tautomer ones - due to an Excited State Intramolecular Proton Transfer (ESIPT). Both forms fluoresce at different wavelengths and thus generate a dual emission proving sensitive to H-bonding and electric field of the environment for which the intensity ratio of the two bands can be used as the reporting unit. In this context, we first reported push-pull probes with advanced features, made from $\mathbf{d U}$ and $\mathbf{3 H C}$. These probes were characterized by a two-color emission (cyan and yellow), good quantum yields in different solvents including water and especially high sensitivity to hydration and NA conformations ${ }^{[9,10]}$. The possibility to connect the $\mathbf{3 H C}$ moiety to adenine nucleobases for NA labeling was next explored by electronically coupling 7-deazadeoxyadenosine (d7A) with 3HC dyes (Figure 1: FCA and TCA) ${ }^{[11]}$. The new conjugates showed several improvements in the photophysical properties of 3HC as evidenced by red-shifted absorptions from UV to the violet range, up to 1.5-fold increase in absorptivity and strong sensitivity to environmental changes. However, they revealed to be strongly quenched in polar protic solvents and almost non-emissive in water. Thus, the access of fluorescent purine-based nucleosides for NA labeling turned out to be more problematic than for the pyrimidine nucleobase. Since 7dA is a potent donor for Photoinduced Electron Transfer (PET), we hypothesized that a proton coupled (PET) ${ }^{[12]}$ from the 7dA fragment to the $\mathbf{3 H C}$ could be a plausible mechanism to account for fluorescence quenching of FCA and TCA. Proposing such a process also raises the question of whether the electron transfer is intramolecular or intermolecular?

It is often difficult to prove the mechanism of quenching. The mechanism may not be exclusive and can be a combination of different processes (e.g. internal conversions, Dexter energy transfer, Twisted Internal Charge Transfer (TICT) conformation) making the task even more difficult ${ }^{[13]}$. To have more convincing experimental evidences, we carried out several experiments with 3-MethoxyChromones (3MCs) to avoid spectroscopic and kinetic complications due to the ESIPT reaction (Figure 1). Herein, we report on our investigation of push-pull systems between dA analogs and 3MCs. First, the energetic aspect of the PET reaction was studied between the separated d7A electron donor and 3MC electron acceptor using cyclic voltammetry to demonstrate whether $\mathbf{~} 7 \mathbf{A}$ can be oxidized and $\mathbf{3 M C}$ reduced. Given that $\mathbf{d G}$ has the lowest oxidation potential 
$\mathrm{E}$ and that incorporation of an $N$ atom at position 8 of the purine increases this value $(\mathrm{E}(\mathbf{d G}) \approx$ $\mathrm{E}(\mathbf{7 d A})<\mathrm{E}(\mathbf{8 d 7 A}) \approx \mathrm{E}(\mathbf{d A}))^{[14-16]}$, we compared with positive $(\mathbf{d G})$ and negative controls $(\mathbf{d A}$ and 8d7A (8-aza-7-deaza-2'-deoxyadenosine)). Secondly, the photophysical investigations of the single-band emissive conjugates (MFCA and MTCA) and their corresponding negative controls (MFC8A, MTC8A and acidification) were performed to verify quenching of MFCA and MTCA in polar protic solvents. Lastly, UV-Vis transient absorption (TA) spectroscopy was performed on the conjugates to establish direct evidence of PET for MFCA and MTCA in MeOH. This study not only provides a better understanding of the PET quenching reaction in separated as well as conjugated systems of $\mathbf{d A}$ analogs and chromones but also brings useful information for the design of advanced fluorescent DNA sensors. Noticeably, fluorescence could be switched ON/OFF by using the suitable $\mathbf{d A}$ analog or simply by fine tuning the medium $\mathrm{pH}$.

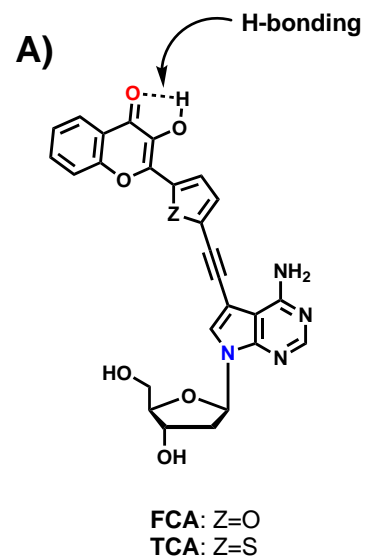

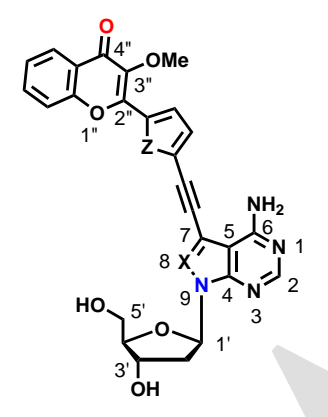

MFCA: $X=\mathrm{CH}, \mathrm{Z}=\mathrm{O}$ MTCA: $X=\mathrm{CH}, \mathrm{Z}=\mathrm{S}$ MFC8A: $X=N, Z=O$ MTC8A: $X=N, Z=S$

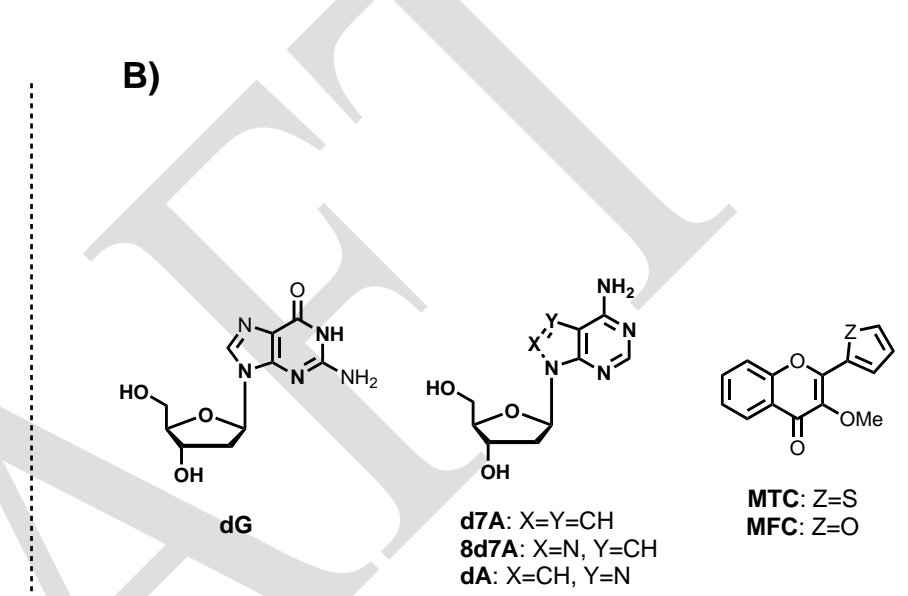

Figure 1. A) Synthesized push-pull conjugated systems investigated here: electron donors and acceptors are depicted in blue and red, respectively. B) Structures of separated donors ( $\mathbf{A G}, \mathbf{d A}$ and derivatives) and acceptors (3MC derivatives).

\section{Results and discussion}

\section{Separated donors and acceptors, and conjugated deoxyadenosine analogs.}

The compounds required for the photophysics were synthesized (MTCA, MFCA, MTC8A, and MFC8A) or obtained from commercial sources (MFC, dG, d7A, 8d7A, and dA). The synthesis of MTC was recently reported by our group ${ }^{[17]}$. Synthesis of conjugates were adapted from Seela's previous works ${ }^{[15,18]}$. The full details of their preparations and characterization are given in SI.

\section{Standard free energy changes of PET reaction between separated donors and acceptors}

PET is the most common mechanism proposed to account for quenching of fluorophores by nucleobases ${ }^{[8]}$. Upon PET, the $\mathbf{3 M C}$ fluorophore can be reduced by the nucleobase to form a radical ion pair, which can further undergo radiationless relaxation to the ground state. Comparison of the redox potentials of the fluorophore and nucleobase allows us to examine the possibility for PET ${ }^{[14]}$. Among the canonical nucleobases, guanine is the most potent electron donor with the lowest oxidation potential ${ }^{[14,19]}$. The redox potential of $\mathbf{7 d \mathbf { A }}$, a prospective nucleobase moiety to engineer adenosine-based probes, is close to that of guanosine ${ }^{[14]}$. One strategy to raise the oxidation potential of $\mathbf{7 d A}$ is to incorporate an endocyclic nitrogen in position 8 of the purine 
framework. Seela et al. exploited this approach and demonstrated its efficiency to affect the oxidation potential, placing $\mathbf{8} \mathbf{d} 7 \mathbf{A}$ in the range comparable to $\mathbf{d} \mathbf{A}^{[15]}$.

To determine if PET is energetically favorable, we have correlated fluorescence quenching of the bridged donor-acceptor systems with the redox potentials of the separated donors and acceptors and their corresponding Gibbs energies calculated from the Rehm-Weller equation (Eq. 1) ${ }^{[13,20]}$. Based on the latter, one could predict whether the electron transfer process is exergonic or endergonic.

$$
\Delta G_{e t}^{\circ}=E^{o x}(D)-E^{r e d}(A)-E_{00}(A)+\Delta G^{\circ}(\varepsilon)
$$

Where:

$\mathrm{E}^{\mathrm{ox}}(\mathrm{D})$ is the oxidation potential of the donor (purine base)

$\mathrm{E}^{\mathrm{red}}(\mathrm{A})$ is the reduction potential of acceptor (3MC derivatives)

$E_{00}(A)$ is the zero-zero transition energy $(1240 / \lambda(\mathrm{nm})$ in $\mathrm{eV})$, where $\lambda$ is the intersection of the normalized absorption and emission spectra of the acceptor ${ }^{[13]}$.

$\Delta \mathrm{G}^{\circ}(\varepsilon)$ is the solvent term contribution and depends on the dielectric constant of the solvent. It was estimated to be $0.1 \mathrm{eV}$ in $\mathrm{H}_{2} \mathrm{O}^{[14]}$.

In this study, we assumed that $\mathbf{d A}$ analogs (d7A and 8d7A) being the electron donors and 3MCs being the electron acceptors (Figure 1B). In order to compare with $\mathbf{d A}$ analogs, natural $\mathbf{d G}$ and dA were investigated as positive and negative controls, respectively ${ }^{[19]}$. Oxidation and reduction potentials, as well as calculated Gibbs energies are reported in Table 1.

Table 1. Oxidation potentials of donors, reduction potentials of acceptors and Gibbs energies

\begin{tabular}{cccccc}
\hline Donors & Acceptors & $\begin{array}{c}\mathrm{E}^{\mathrm{ox}}(\mathrm{D})^{[\mathrm{a}]} \\
v s \mathrm{NHE} \\
{[\mathrm{V}]}\end{array}$ & $\begin{array}{c}\mathrm{E}^{\mathrm{red}}(\mathrm{A})^{\mathrm{a}} \\
v s \mathrm{NHE} \\
{[\mathrm{V}]}\end{array}$ & $\begin{array}{c}\mathrm{E}_{00}(\mathrm{~A})^{\mathrm{b}} \\
{[\mathrm{eV}]}\end{array}$ & $\begin{array}{c}\Delta \mathrm{G}_{\text {et }}^{\circ} \\
{[\mathrm{eV}]}\end{array}$ \\
\hline $\mathbf{d G}$ & $\mathbf{M T C}$ & +1.42 & -1.50 & 3.16 & -0.33 \\
$\mathbf{d 7 A}$ & $\mathbf{M T C}$ & +1.46 & -1.50 & 3.16 & -0.30 \\
$\mathbf{8 d 7 A}$ & $\mathbf{M T C}$ & +1.81 & -1.50 & 3.16 & 0.05 \\
$\mathbf{d A}$ & $\mathbf{M T C}$ & +1.85 & -1.50 & 3.16 & 0.10 \\
$\mathbf{d G}$ & $\mathbf{M F C}$ & +1.42 & -1.50 & 3.18 & -0.33 \\
$\mathbf{d 7 A}$ & $\mathbf{M F C}$ & +1.46 & -1.50 & 3.18 & -0.30 \\
$\mathbf{8 d 7 A}$ & $\mathbf{M F C}$ & +1.81 & -1.50 & 3.18 & 0.05 \\
$\mathbf{d A}$ & $\mathbf{M F C}$ & +1.85 & -1.50 & 3.18 & 0.10 \\
\hline
\end{tabular}

[a] Cyclic voltammetry was conducted against $\mathrm{Ag} / \mathrm{AgCl}(\mathrm{KCl}$ saturated) in acetonitrile containing $1 \mathrm{M}$ tetra- $n$ butylammonium hexafluorophosphate $\left(\mathrm{TBAPF}_{6}\right)$ as the electrolyte, and saturated solution of the purine base or $1 \mathrm{mM}$ 3MC. Data were converted into normal hydrogen electrode (NHE) for comparison. Scan speed was $100 \mathrm{mV} / \mathrm{s}$. [b] $\mathrm{E}_{00}(\mathrm{~A})$ values were obtained from absorption and emission spectra of the corresponding fluorophore in water (See Figure S2).

Analysis of these data allows several conclusions to be drawn. First, the oxidation potentials obtained for the known purine compounds were comparable to the reported values highlighting the consistency of our data (e.g. compare $+1.46 \mathrm{~V}$ with $+1.48 \mathrm{~V}$ for $\mathbf{d 7 A}$, for the other compounds see Table S2) ${ }^{[15]}$. The oxidation potential of $\mathbf{d 7 A}(+1.46 \mathrm{~V})$ is close to that of $\mathbf{d G}(+1.42 \mathrm{~V})$; 
similarly, 8d7A $(+1.81 \mathrm{~V})$ is comparable to $\mathbf{d A}(+1.85 \mathrm{~V})$. According to the Rehm-Weller equation, $\Delta G^{\circ}{ }_{e t}$ depends on three main parameters. Firstly, it depends on the oxidation and reduction potentials of the donor and acceptor, respectively. Table 1 shows that the reduction potentials of the two chromones are identical; by contrast, the oxidation potentials of the purine bases are discriminant (compare $\mathbf{~} 7 \mathbf{A} v s$. dG with 8d7A $v s . \mathbf{d A}$ ). Hence, $\Delta G^{\circ}{ }_{e t}$ of any pair between donor (d7A or dG) and acceptor (MTC or MFC) will be about $0.35-0.43 \mathrm{eV}$ smaller than that of 8d7A or $\mathbf{~ d A ~ a s ~ t h e ~ d o n o r . ~ B a s i c a l l y , ~ o x i d a t i o n ~ o f ~ t h e ~ d o n o r ~ a n d ~ r e d u c t i o n ~ o f ~ t h e ~ a c c e p t o r ~ i n ~ a ~ P E T ~}$ process requires energy. Therefore, this energy contributes a positive value to the $\Delta G^{o}{ }_{e t}$ of the PET reaction. Secondly, $\Delta G_{e t}^{o}$ depends on the zero-zero transition energy $\left(\mathrm{E}_{00}\right)$ of the fluorophore (3MC). $\mathrm{E}_{00}$ is the energy gain of the photoexcited $\mathbf{3 M C}$, it contributes a negative value to the free energy. The zero-zero transition energy of the furyl chromone (MFC, $3.18 \mathrm{eV}$ ) is just slightly higher than that of thiophene-containing chromone (MTC, 3.16 V). This result is consistent with the fact that the larger sulfur atom probably decreases of the HOMO-LUMO energy gap compared to the smaller oxygen atom due to resonance effects ${ }^{[21,22]}$. Lastly, the solvent term contribution $\Delta \mathrm{G}^{\circ}(\varepsilon)$ depends on the dielectric constant of the solvent. Generally, it decreases with increasing permittivity; however, its contribution to the total energy is small. According to $\Delta G^{o}{ }_{e}$, electron transfer from d7A to MFC and MTC is exergonic with $-0.30 \mathrm{eV}$; while, it is slightly endergonic from 8d7A to the chromones MTC and MFC with $+0.05 \mathrm{eV}$. Assuming that $\Delta G^{\circ}{ }_{e t}=-0.30 \mathrm{eV}$ is in the normal region according to the Marcus theory ${ }^{[23-25]}$, our results suggest that the electron transfer from d7A to the chromone should be faster and more favorable than that from 8d7A.

\section{General photophysical characterization}

The photophysics of the newly synthesized push-pull nucleosides were characterized in a set of four protic solvents (water, methanol, ethanol, and $i$-propanol) of different polarities, and the results are summarized in Table 2. The Dimroth-Reichardt polarity index parameter ${ }^{[26]}$ is used to rank the solvents. This empirical scale takes into account the dielectric constant and H-bonding ability of the solvent. The acidity according to the scale is also given for comparison and discussion [27]. 
Table 2. Photophysical properties of the nucleoside analogs in different solvents.

\begin{tabular}{|c|c|c|c|c|c|c|}
\hline \multicolumn{2}{|c|}{$\varepsilon_{\max }\left(10^{3} \mathrm{M}^{-1} \cdot \mathrm{cm}^{-1}\right)^{[\mathrm{a}]}$} & - & 40 & 40 & 34 & 37 \\
\hline Solvent & $\begin{array}{r}E_{\mathrm{T}^{\mathrm{N}}}(30)^{[\mathrm{b}]} \\
S A^{[\mathrm{c}]}\end{array}$ & $\begin{array}{c}\lambda \\
(\mathrm{nm}),{ }^{[\mathrm{d}]} \\
\Phi \\
(\%)^{[\mathrm{e}]}\end{array}$ & $\begin{array}{c}\text { MFCA } \\
\text { (10) }\end{array}$ & $\begin{array}{c}\text { MTCA } \\
\text { (11) }\end{array}$ & $\begin{array}{c}\text { MFC8A } \\
\text { (12) }\end{array}$ & $\begin{array}{c}\text { MTC8 } \\
\text { A } \\
(13)\end{array}$ \\
\hline \multirow[t]{3}{*}{$\overline{\mathrm{H}_{2} \mathrm{O}^{[\mathrm{f}]}}$} & 1.00 & $\bar{\lambda} \lambda$ & 378 & 388 & 372 & 366 \\
\hline & 1.062 & $\lambda_{\mathrm{Em}}$ & 567 & 579 & 472 & 479 \\
\hline & & $\Phi$ & 0.2 & 0.7 & 30 & $\#^{N A}{ }^{[\mathrm{g}]}$ \\
\hline \multirow[t]{3}{*}{$\begin{array}{l}\mathrm{H}_{2} \mathrm{O}-\mathrm{TFA} \\
5 \%{ }^{[\mathrm{f}]}\end{array}$} & & $\lambda_{\mathrm{Abs}}$ & 377 & 384 & & \\
\hline & & $\lambda_{\mathrm{Em}}$ & 521 & 527 & & \\
\hline & & & & & & \\
\hline \multirow[t]{3}{*}{$\mathrm{MeOH}^{[\mathrm{f}]}$} & 0.76 & $\lambda_{\mathrm{Abs}}$ & 378 & 388 & 372 & 366 \\
\hline & 0.605 & $\lambda_{\mathrm{Em}}$ & 571 & 580 & 464 & 469 \\
\hline & & $\Phi$ & 1.3 & 0.9 & 38 & 36 \\
\hline $\mathrm{MeOH}^{[\mathrm{h}]}$ & & $\lambda_{\mathrm{Em}}$ & 512 & 512 & 464 & 469 \\
\hline \multirow[t]{3}{*}{$\begin{array}{l}\text { MeOH - } \\
\text { TFA 5\% }{ }^{[\mathrm{f}]}\end{array}$} & & $\lambda_{\mathrm{Abs}}$ & & 382 & & \\
\hline & & $\lambda_{\mathrm{Em}}$ & 501 & 508 & & \\
\hline & & $\Phi$ & 41 & 35 & & \\
\hline \multirow[t]{3}{*}{$\mathrm{EtOH}^{[\mathrm{f}]}$} & 0.65 & $\lambda_{\mathrm{Abs}}$ & 378 & 390 & 368 & 379 \\
\hline & 0.400 & $\lambda_{\mathrm{Em}}$ & 555 & 564 & 458 & 464 \\
\hline & & $\Phi$ & 6 & 4.2 & 33 & 30 \\
\hline \multirow[t]{3}{*}{$i$-PrOH ${ }^{[\mathrm{f}]}$} & 0.57 & $\lambda_{\mathrm{Abs}}$ & 379 & 391 & 368 & 379 \\
\hline & 0.283 & $\lambda_{\mathrm{Em}}$ & 534 & 546 & 453 & 459 \\
\hline & & $\Phi$ & 19 & 18 & 29 & 26 \\
\hline
\end{tabular}

[a] Molar extinction coefficient was determined in methanol; relative standard deviations are lower or equal to $5 \%$. [b] Reichardt's empirical solvent polarity index ${ }^{[26]}$. [c] Acidy scale (ref ${ }^{[27]}$ ). [d] Wavelength of the absorption maximum $\left(\lambda_{\mathrm{Abs}}\right)$ and emission maximum $\left(\lambda_{\mathrm{Em}}\right)$ in $\mathrm{nm}$. [e] $p$-DiMethylAminoFlavone (DMAF) in EtOH $\left(\lambda_{\mathrm{Abs}}=404\right.$ $\mathrm{nm}, \Phi=27 \%)^{[28]}$ was used as the standard reference Fluorescence quantum yields $(\Phi)$. [f] Absorption and emission spectra were recorded at concentrations of $11 \mu \mathrm{M}$ and $1.1 \mu \mathrm{M}$, respectively. [g] Not investigated due to very low solubility. [h] Emission maximum obtained for solution at concentration $<10 \mathrm{nM}$. 

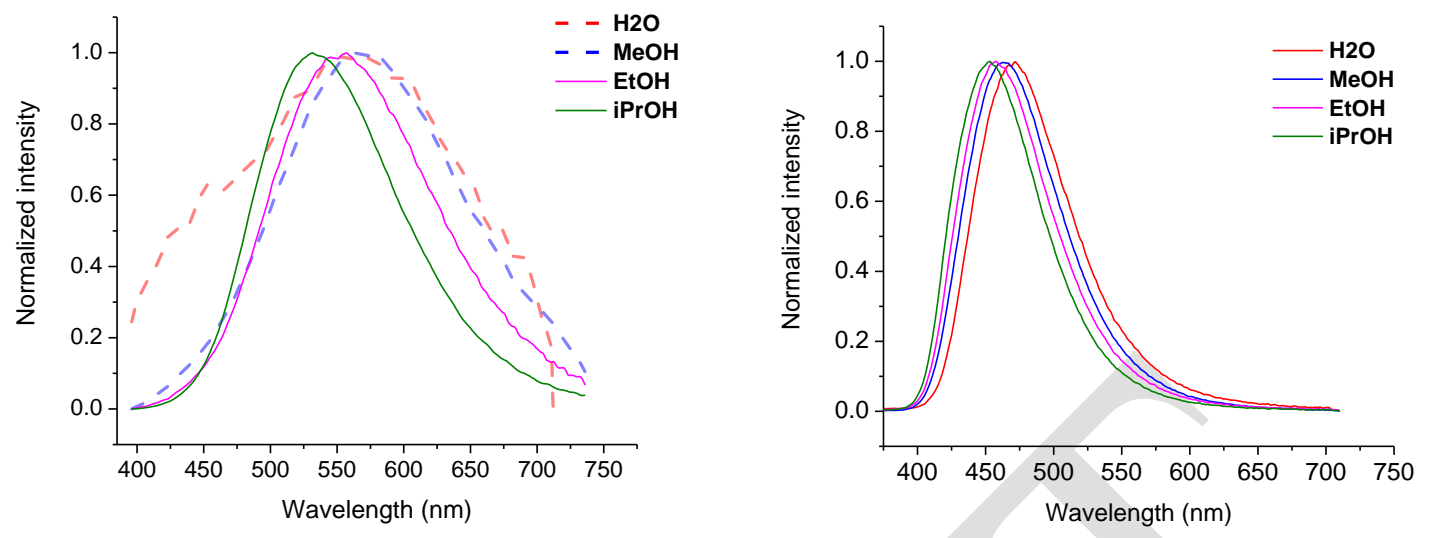

Figure 2. Normalized emission spectra of MFCA (left) and MFC8A (right) in protic solvents (at $1.1 \mu \mathrm{M})$ of increasing polarity. Excitation wavelength was at the maximum of absorption (Table 2).
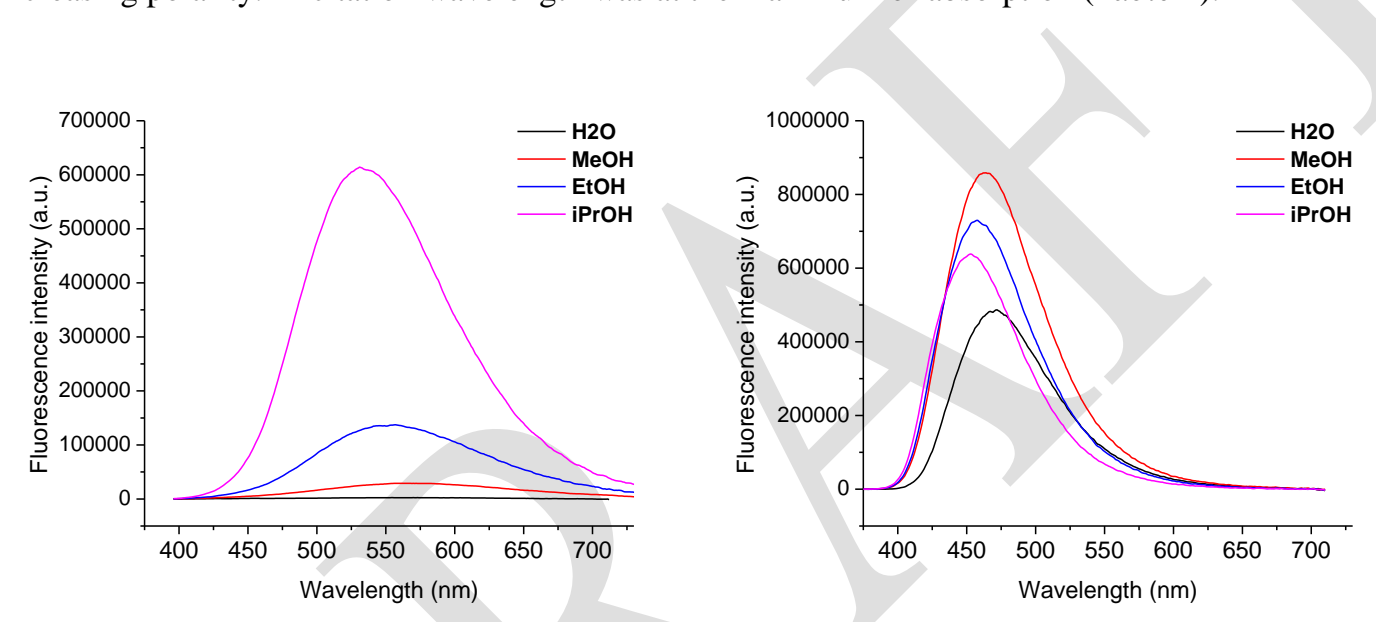

Figure 3. Fluorescence spectra of MFCA (left) and MFC8A (right) in protic solvents (at 1.1 $\mu \mathrm{M}$ ). Excitation wavelength was at the maximum of absorption (Table 2).

The MFCA and MTCA exhibit similar absorptivity and little variation of absorption maxima whatever the solvent studied. The maxima are centered near the visible range (377-379 and 388$391 \mathrm{~nm}$, respectively). Both conjugates displayed a 35-41 nm bathochromic shift of their absorption by comparison to the parent MFC and MTC chromones (Figure $S 2$ and ref ${ }^{[17]}$ ) as expected for dyes with extended conjugation. The same conclusion can be deduced from the UVVisible spectra of MFC8A and MTC8A, albeit with slightly blue-shifted absorption maxima (362-383 nm). As expected, substitution of the 3-hydroxy proton by the methyl group in the chromone inhibits ESIPT and thus, the dual emission. MFCA and MTCA displayed bathochromic shifts of their emission maxima with the increase in solvent polarity (e.g. from $534 \mathrm{~nm}$ in $i$-PrOH to $571 \mathrm{~nm}$ in $\mathrm{MeOH}$ for MFCA, see Table 2), as illustrated in Figure 2. Their quantum yields gradually drop as the polarity increases (Figure 3). For chromones, it is known that in the excited state, the electron-enriched 4" carbonyl oxygen (Figure 1) is particularly sensitive to H-bonding for which $\mathrm{H}$-bond interactions are all the stronger as the acidity of the solvent increase ${ }^{[29,30]}$. There are many examples in chemistry and biology supporting that intermolecular site-specific 
interactions through H-bonding can increase PET ${ }^{[12,14,31]}$. As with FCA and TCA compared to TCU, the corresponding methylated derivatives behave differently. They turn almost non-emissive in $\mathrm{MeOH}$ and $\mathrm{H}_{2} \mathrm{O}$ (e.g. compare $\Phi=0.9 \%$ for MTCA with $\Phi=49 \%$, in $\mathrm{MeOH}$ ) and show larger bathochromic shifts, which can be seen from the shifts in emission since the compounds have close absorption maxima (e.g. compare $501 \mathrm{~nm}$ for MTCU with $580 \mathrm{~nm}$ for MTCA in MeOH).

Why do these compounds demonstrate such large spectral shifts and drop in quantum yields in protic solvents? Are these variations due to solvent specific effects, conformational changes generating a TICT conformer or probe-probe interactions? To address this question, we examined the emission spectra recorded at different concentrations ranging from $60 \mu \mathrm{M}$ to $1.25 \mathrm{nM}$ as shown in Figure 4 (see also Figure S8), and observed concentration dependent spectral shifts.
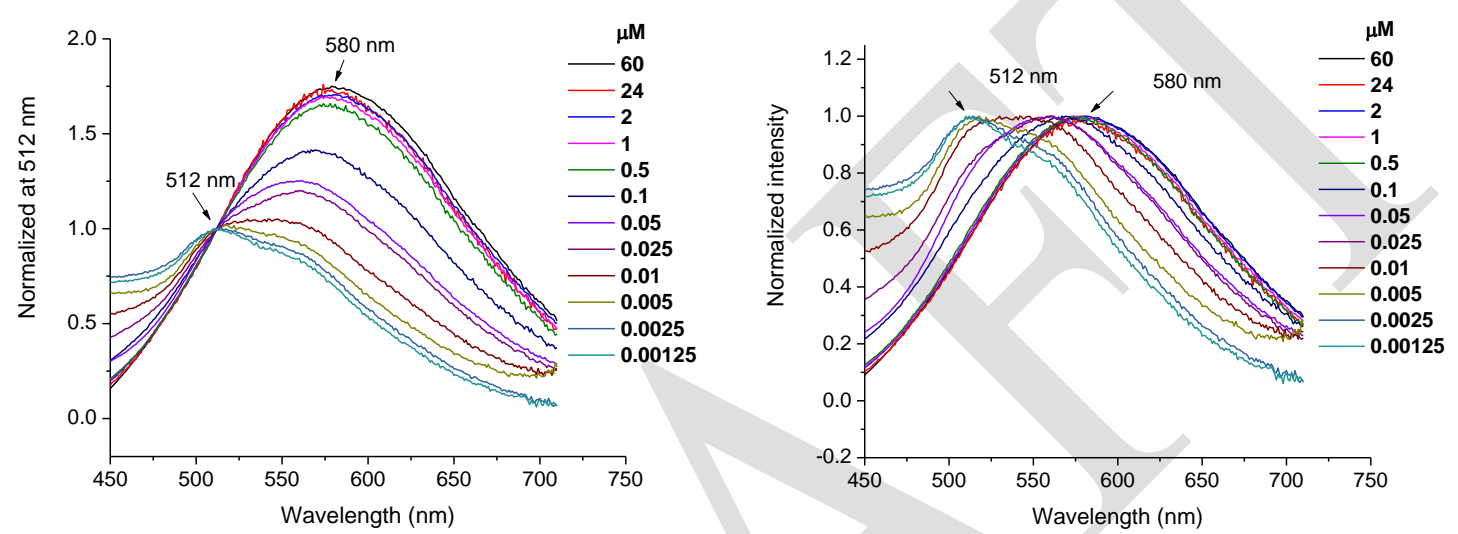

Figure 4. Normalized emission spectra of MTCA at $512 \mathrm{~nm}$ (left) and at the maximum (right) at different concentrations in $\mathrm{MeOH}$ (excitation wavelength at $370 \mathrm{~nm}$ ).

For example, the emission maximum of MTCA in MeOH was gradually blue-shifted from 580 $\mathrm{nm}$ at concentrations larger than $0.5 \mu \mathrm{M}$ to $512 \mathrm{~nm}$ below $2 \mathrm{nM}$. At concentrations as low as $5 \mathrm{nM}$, we have already detected a contribution of the 580-nm emissive species (see Figure 4-left). The high-energy emission band was close to that of MTCU. We therefore attribute the $512 \mathrm{~nm}$ emission band to the monomer dye, and that at $580 \mathrm{~nm}$ to a complex. Excimers are typically observed as a broad emission band red shifted relative to that of the monomer fluorophore. Excimer is a complex formed between two monomers when at least one component is in the excited state. Noticeably, the changes in the emission spectra of MTCA and MFCA clearly showed that the complexes are formed at concentrations as low as few $\mathrm{nM}$, which are far below the $\mathrm{mM}$ concentration range for which excimers are typically observed ${ }^{[13,32]}$. Indeed at $2 \mu \mathrm{M}$ concentration, the average distance between solute molecules of $\mathrm{d} \sim 0.1 \mu \mathrm{m}$ corresponds to a typical diffusion time $\tau=\mathrm{d}^{2} / \mathrm{D}$ in the $20 \mu \mathrm{s}$ range for two molecules to encounter (assuming $\mathrm{D}=500 \mu \mathrm{m}^{2} / \mathrm{s}$ for the diffusion coefficient ${ }^{[33,34]}$, which is 4 orders of magnitude longer than the excited state lifetime. Thus, the emission spectrum observed at concentrations of $0.5 \mu \mathrm{M}$ or larger is attributed to a complex resulting from ground state interactions. Analyzing the excitation spectra recorded at different concentrations further supports this interpretation as the existence of two absorbing species was evidenced at low concentration (Figures S7 and S8). Formation of ground-state complexes of acceptor/donor pairs is known ${ }^{[35,36]}$. The close association of both species favors photoinduced electron transfer generating radical ion pairs. The radical ion pair generated from the charge transfer complex can be compared to the well-known poorly emissive geminate radical 
ion pairs for exciplexes, although the later, as for the excimer, generally refer to bimolecular contacts of the excited dye and a quencher ${ }^{[37-40]}$. What is particular to our compounds is that they formed complexes even at very low concentrations. We are not aware of other examples of such intermolecular tight interactions reported in the literature. In the case of MTCA and MFCA, their complexation could be favored in polar solvents due to the hydrophobic nature of the extended flat fluorophore, favorable stacking and van der Waals interactions as well as a quadrupole arrangement allowing strong coulombic coupling. An head-to-tail orientation of the two dyes should favor intermolecular PET, because the nucleobase donor in one species would be in contact with the chromone acceptor of the other species (Figure 5). Thus, the complexation of MTCA and MFCA and photo-induced formation of poorly emissive radical ion pairs likely dominate the photophysics of these dyes in polar protic solvents.

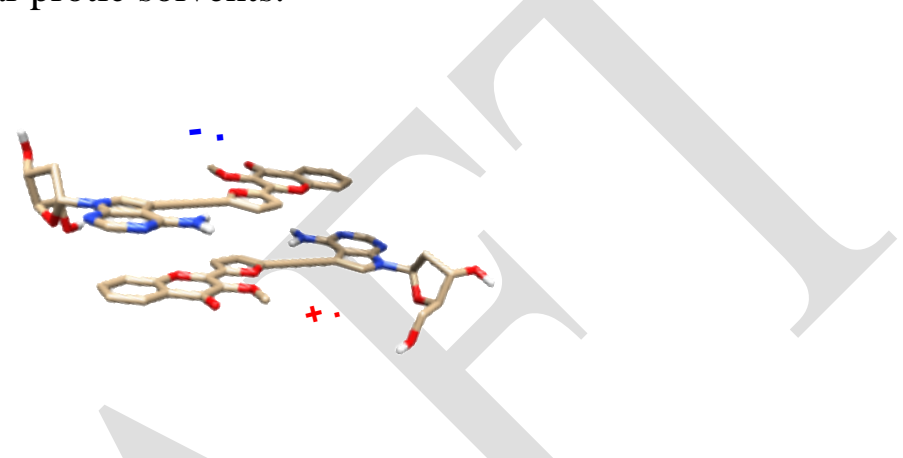

Figure 5. Proposed representation of the complexed, radical pair structure (Photo generated from Chimera)

The 8-aza series (MTC8A and MFC8A) behaves differently. They showed reduced redshifts of their emission maxima along with the increase in solvent polarity (e.g. from $453 \mathrm{~nm}$ in $i-\mathrm{PrOH}$ to $474 \mathrm{~nm}$ in $\mathrm{MeOH}$ for MFC8A) and bright emissions in polar protic solvents (e.g. 30\% in water for MFC8A). Importantly, the emission spectra remain unchanged even down to the 1-2 $\mathrm{nM}$ range for both dyes by contrast to MTCA and MFCA (Figures S11 and S12). These results suggest that the MTC8A and MFC8A were observed as monomers in MeOH solution at all studied concentrations from $\mathrm{nM}$ to $\mu \mathrm{M}$. Since the two types of dyes have the same size and area, the only difference between them is the introduction of an electron-withdrawing nitrogen at the 8 position on the nucleobase. This considerably reduces the electron-donating ability of $\mathbf{8 d 7} \mathbf{A}$ as evidenced by the increased of the oxidation potential (Table 1, compare 1.81 and $1.46 \mathrm{~V}$ for $8 \mathbf{d 7 A}$ and $\mathbf{d 7 A}$, respectively). Therefore, the difference between the oxidation potential of the donor and the reduction potential of the acceptor increases. As a consequence, the propensity to form charge transfer complexes between the 8d7A donor and the MTC or MFC acceptor should be greatly affected by contrast to that formed with $\mathbf{d 7 A}$. Previous reports have shown that the association constant of donor/acceptor complexes decreases as the difference in the redox potential decreases ${ }^{[35,36]}$, albeit they form at concentrations several orders of magnitude higher than those required for MFCA and MTCA. Our data highlight that the difference in the redox potential is one important contributor to the complex association for our dyes. 


\section{Acidic-mediated inhibition of PET process}

Protonation of amines linked to weakly emissive systems is a common way to switch on their fluorescence ${ }^{[41]}$. The purine bases have 2 electron-rich centers at $N 3$ and $N 1$ atoms with $\mathrm{p} K_{\mathrm{a}}$ of around $4.3^{[42]}$. In acidic media, the most basic nitrogen $N 1$ will be protonated. Protonation of the purine base should therefore reduce its electron-donating ability and disrupt both the complex formation and electron transfer process (Figure 6). To check this hypothesis, the absorption and emission spectra of MFCA and MTCA in $\mathrm{MeOH}$ and $\mathrm{H}_{2} \mathrm{O}$, with and without adding TFA $(0.5 \%$ $v / v)$, were investigated. Upon addition of this strong Brønsted acid, turn-on emissions were recorded (Figures 6 and S13). For instance, the poor quantum yields $(<1 \%)$ of MTCA increased to $35 \%$ and $6 \%$ for acidified $\mathrm{MeOH}$ and $\mathrm{H}_{2} \mathrm{O}$ solutions, respectively. Analysis of the excitation and emission spectra of MFCA and MTCA in acidified MeOH (TFA, 0.5\% v/v) at different concentrations leads to the same conclusion as for MTC8A and MFC8A (Figures S9 and S10), that only the monomer spectra are observed at all concentrations used here.
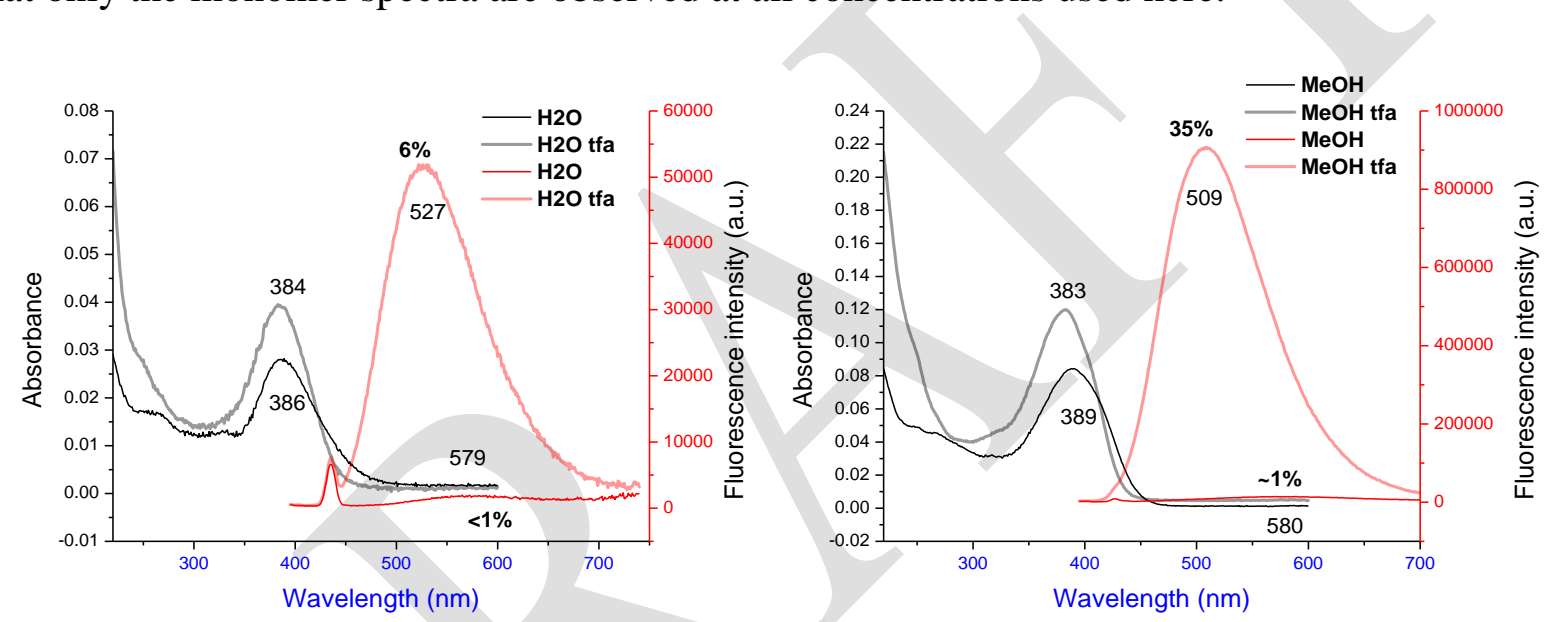

Figure 6. Substantial fluorescent turn-on signal of MTCA upon TFA addition (absorption spectra at $11 \mu \mathrm{M}$ (left) and $33 \mu \mathrm{M}$ (right) in grey and black, emission spectra at $2 \mu \mathrm{M}$ in red and rose. Absorption and emission maxima are given in $\mathrm{nm}$ and quantum yields in\% above the emission maxima (determination of quantum yields took into account the change in absorbance at the excitation wavelength at $370 \mathrm{~nm}$ ).

\section{Direct observation of PET on conjugated system using UV-Vis TA spectroscopy}

To further support the proposed PET reaction and observe the corresponding reaction kinetics, we used femtosecond UV-Vis transient absorption (TA) spectroscopy. Methanolic solutions of MTCA, MTC8A and MTCA with TFA $(5 \%, v / v)$ - as well as MFCA, MFC8A and MFCA with TFA $(5 \% v / v)$ - were prepared at a concentration of $330 \mu \mathrm{M}$ (i.e. absorbance $\mathrm{A}=1 / \mathrm{mm}$ at $\lambda_{\max }$ ) and investigated by TA, with a 60 to $70 \mathrm{fs}$ time resolution, using a 370-nm pump pulse, and a white-light probe pulse offering an absorption detection window spanning from 330 to $700 \mathrm{~nm}$. The experimental set-up is the same as previously described ${ }^{[43]}$ (see also SI). Quantitative analysis of the TA data is performed by global analysis (see SI) and illustrated by presenting the so-called decay-associated spectra (DAS, shown in the SI), which reveal the time scales and associated spectral modifications characterizing the TA decay kinetics recorded across the entire UV-Vis observation window. 

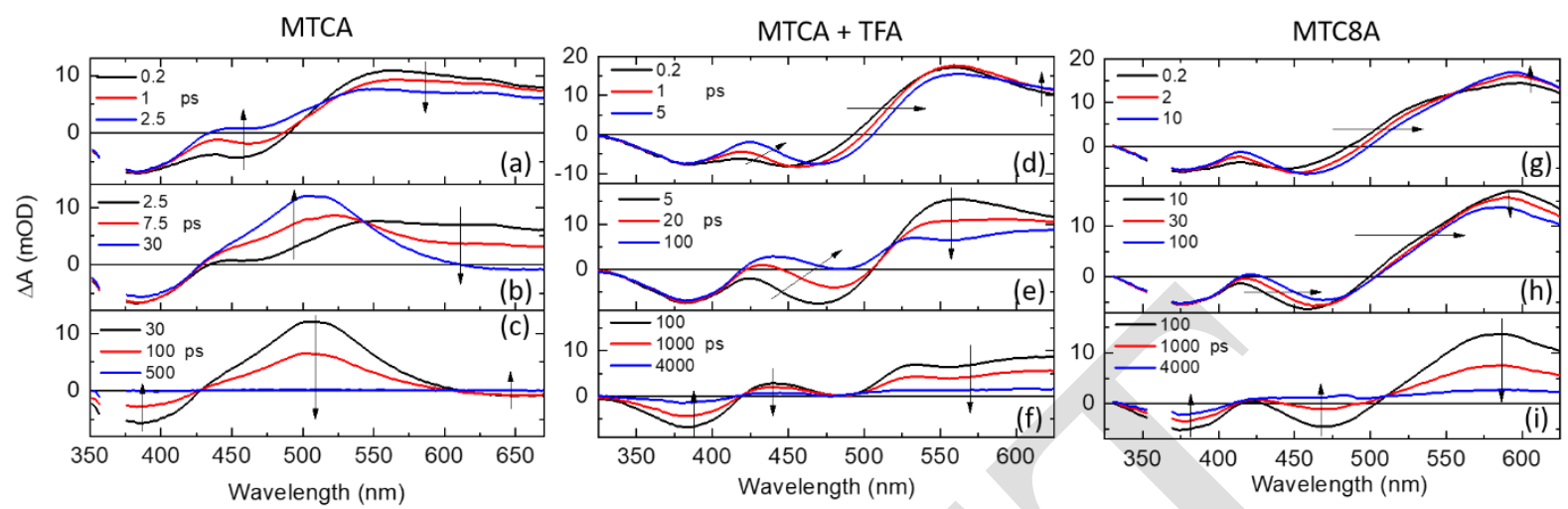

Figure 7. TA data $(\triangle \mathrm{A})$ measured upon $370 \mathrm{~nm}$ excitation of methanol solutions of MTCA (left), MTCA+TFA (middle), and MTC8A (right). A selection of transient spectra at early (first line: panels a,d,g), intermediate (second line: panels b, e, h) and later pump-probe time delays (third line: panels c, f, i) are displayed to illustrate the spectral evolutions observed on various times scales and highlighted by the black arrows.

Immediately after excitation, the early signature of the first excited state $S_{1}$ is similar in the three samples (0.2 ps spectra in Figure 7, three panels of the first line). We identify the ground-state bleach (GSB) as a negative signal around $380 \mathrm{~nm}$, a stimulated emission (SE) band - also a negative signal - around $450 \mathrm{~nm}$, and a positive excited state absorption at wavelengths > $500 \mathrm{~nm}$. Early spectral relaxation occurs in all three samples already within the instrument response function, and with a 0.5 to 0.6 ps time scale (see Supplementary Figure S16), likely due to fast solvent relaxation [44] and possibly to early vibrational relaxation out of the Franck Condon region. Then, in the case of MTCA, the early SE and ESA band decay on the 2.8 ps time scale, corresponding to the formation of a first transient state that we name $\mathbf{U}$, which is still and excited state (possibly the MTCA complex in a different conformation), since the GSB does not recover on this time scale (Figure 7 panels a and b). This state then decays on the $12 \mathrm{ps}$ time scale to form a new state, that we name $\mathbf{V}$, characterized by an intense absorption band around $510 \mathrm{~nm}$ and a new, weak (negative) SE band at $\lambda>610 \mathrm{~nm}$ (see the 30 -ps spectrum in panel b), while still hardly any recovery of the GSB signal occurs. The $\mathbf{V}$ state then decays with a 100-ps time scale, with the TA signal decaying completely to zero while keeping the same shape (see panel c), thus indicating the reformation of the original ground state on this time scale. The two successive SE bands observed here - i.e. at $450 \mathrm{~nm}$ with a $2.8 \mathrm{ps}$ lifetime, and $>610 \mathrm{~nm}$ with a 100 -ps lifetime - must be closely related to the emission spectrum displayed for the same compound in Figure S4. In the observed spectroscopic signatures of the transient $\mathbf{V}$ state, the intense (positive) 510-nm ESA band overlaps and masks the (negative, less intense) SE band which is expected to be maximum at $580 \mathrm{~nm}$, according to the steady state spectrum (see Table 2). Due to this spectral overlap and larger intensity of the ESA signal, we actually only observe the red tail of the $\mathbf{V}$ emission in the TA data. The 450-nm SE band of the initially populated $S_{1}$ state is very short-lived and its contribution to the steady-state emission spectrum - expected in its high-energy tail - is strongly quenched.

In the case of MTC8A, following the early, sub-ps kinetics attributed to solvation and early relaxation away from the FC region, the SE and ESA bands both red-shift with a 7.0 ps time constant (see Figure S16). Further redshift of the SE band towards $470 \mathrm{~nm}$ occurs together with a 
slight decay of the ESA band on the 30-ps time scale (Figure S16). This overall multiexponential spectral relaxation is possibly due to structural reorganization of the MTC8A monomer excited state, resulting in the formation of the $469 \mathrm{~nm}$ emissive state detected by steady state emission (see Table 2), that we name U'. Here, the long-lived $470 \mathrm{~nm}$ SE band is not hidden by any overlapping ESA and therefore in perfect agreement with the observed steady-state emission. No strong ESA band at $510 \mathrm{~nm}$ corresponding to the MTCA V state is observed here. The $\mathbf{U}$ ' lifetime is measured to be $1.0 \mathrm{~ns}$ (see Figure S16). Unlike for MTCA, the TA signal of MTC8A does not return to zero within the maximum 5ns time scale achievable in our TA experimental set-up, meaning that the U' state does not decay back to the original ground state, but to a long-lived (>>5ns) photoproduct (PP) state. Specifying the nature of this PP would require complementary investigations and is beyond the scope of this work.

Upon acidification by addition of TFA, the TA signal of MTCA+TFA resembles that of MTC8A, with no intense ESA band at $510 \mathrm{~nm}$, but a multiexponential spectral relaxation, resulting in the red shift of the initial SE band towards $490 \mathrm{~nm}$. The slowest component of this multiexponential relaxation is found to be 23 ps (see Figure S16) and characterizes a decay of the SE and ESA bands while the GSB remains constant. The time scale is similar to that of the formation of the $\mathbf{U}$, intermediate in MTC8A, we therefore also name $\mathbf{U}^{\prime}$ the transient state formed on the 23-ps time scale in MTCA in the presence of TFA. Importantly, MTCA is strongly fluorescent in the presence of TFA (with $\lambda_{\mathrm{em}}=509 \mathrm{~nm}$ and $\Phi=35 \%$, see Table 2). Therefore, we must describe the apparent decay of SE on the 23-ps time scale as the rise of an underlying ESA, leading to a net decay of the TA signal to zero at $480 \mathrm{~nm}$, as a result of two significant but cancelling positive (ESA) and negative (SE) contributions. This emissive U' state is then observed to decay on the 2.2 ns time scale, with no further spectral modification, to reform the original ground state, as indicated by the TA signal decaying fully to zero on this time scale. The same TA data, analysis and interpretation hold for the MFCA, MFC8A and MFCA+TFA methanol solutions as shown in Supplementary Figure S17 and S18.

All together our data are consistent with the following plausible scenario (Figure 8). In polar protic solvents, MTCA or MFCA exist in their ground states most likely as face-to face complexes in head-to-tail relative orientation (vide supra). Light absorption of these complexes generates a first transient $\mathbf{U}$ state within the first $3 p s$ (vibrational relaxation), which rapidly (12ps) leads to the $\mathbf{V}$ state characterized by a strong absorption band at 510nm (MTCA, Figure 7c) or $490 \mathrm{~nm}$ (MFCA, Figure S17) and weak emission band at $580 \mathrm{~nm}$ (MTCA \& MFCA, Table 2) or $571 \mathrm{~nm}$ (MFCA, Table 2). Based on the relative oxydo-reduction potentials and accompanying discussion above, we interpret the $\mathbf{V}$ state as resulting from an intermolecular electron transfer from the $\mathbf{d 7 A}$ donor part of one molecule to the MTC or MFC acceptor part of the other molecule of a ground state preformed complex. On the 100-ps time scale, this geminate radical ion pair would recombine so as to reform to ground state, therefore quenching the already weak 580/571 emission and explaining the very low emission quantum yield $(\sim 1 \%)$ of these species. 


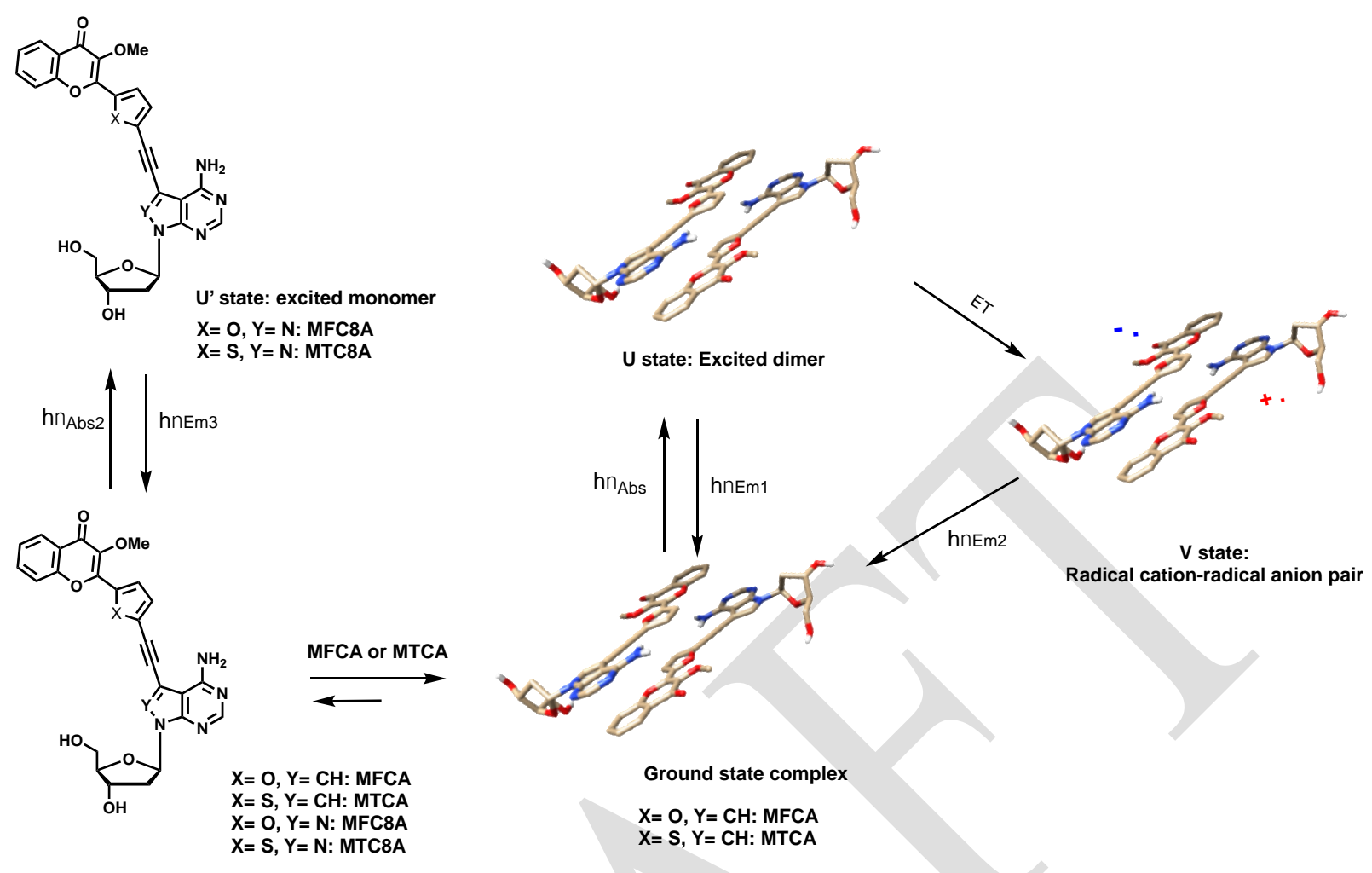

Figure 8. Schematic representation illustrating the formation of ground state complexes favorable only for MFCA and MTCA, PET and radical ion pair products. For clarity, H-bonding with the protic solvent was not presented. The proposed representation of the dimer structure was generated from Chimera $\left(\operatorname{ref}^{[45]}\right)$.

On the other hand, our data did not bring any evidence of the existence of ground state complexes for MTC8A or MFC8A. After excitation of MTC8A or MFC8A, a multiexponential spectral relaxation is assigned to excited state conformational relaxation producing the $\mathbf{U}$ ' excited state characterized an emission spectrum centered at 469/464 nm and a 1-ns-long lifetime in good agreement with a high quantum yield of these nucleoside analogs in $\mathrm{MeOH}$ (36-38\%). Interestingly, acidified solutions of MTCA or MFCA (5\% vol of TFA in MeOH) show results similar to those of MTC8A or MFC8A. A strong spectral relaxation on a comparable time scale yields to a 2-ns-long lived $\mathbf{U}$ ' state emissing fluorescence around $508 / 501 \mathrm{~nm}$, with no sign of the $510 \mathrm{~nm}$ absorption characterizing the $\mathbf{V}$ state in the absence of TFA (Figures 7 and S17). As the introduction of an electron withdrawing nitrogen at position 8, protonation of $\mathbf{d 7 A}$ reduces the electron-rich properties of the nucleobase, which should raise its oxidation potential. As a consequence, no ground state complexation occurs, and the monomer keeps a long life time explaining the quantum yields (35-41\%) of MTCA and MFCA upon acidification with TFA in $\mathrm{MeOH}$ (see Figure 6).

\section{Conclusion}

In the field of fluorescent sensors based on donor-bridge-acceptor fluorophores, intramolecular PET is generally proposed to account for fluorescence quenching ${ }^{[46-48]}$. Our data are consistent 
with a different scenario. MFCA and MTCA likely formed head-to-tail complexes in the ground state in polar protic solvents. Upon excitation, such configuration should be favorable for intermolecular proton coupled PET from the electron donor nucleobase to the electron acceptor chromone. Photoexcitation of these complexes produce a weakly emissive and short-lived state that we assign to a adical ion pair produced by PET, and responsible for the low emission quantum yields. Whether, the monomer is itself quenched by intramolecular PET is a question that remains unanswered because the photophysics of these compounds was dominated by the formation of ground state complexes even at very low concentrations. Will through-bond electron transfer be competitive in this case ${ }^{[47,48]}$ ? Incorporation of MFCA and MTCA into double stranded DNA may help to answer this question because adjacent natural nucleobases should shield the 7dA base from complex formation while exposing the electronically coupled chromone to the major groove and water ${ }^{[49]}$. In the light of our results, it would be interesting to know if formation of poorly emissive charge transfer complexes is particular to our compounds or more common to donor electronically coupled to acceptor ${ }^{[50-52]}$. Answering this question could help reconsidering conjugates that may have been eliminated too quickly as fluorescent probes of biomolecular structures and interactions. On the other hand, protonation of the purine base or introduction of an electron-withdrawing nitrogen at position 8 of the nucleobase are expected to disfavor the putative electron transfer. Certainly, they are observed to prevent complex formation and the corresponding monomers are highly fluorescent. Introduction of the new conjugates into DNA and photophysical characterization of the labeled DNA are currently in progress.

\section{Acknowledgments}

We thank Dr. Sandra Olivero for her support to cyclic voltammetry. We are grateful for the ANR (UCAJEDI project: ANR-15-IDEX-01) and the French Government concerning the PhD grants of H.-N.L and G.B, respectively. This research program was financially supported by the ANR (ANR-12-BS08-0003-02, ANR-15-CE11-0006 "PICO2”, ANR-11-LABX-0058_NIE) and PACA region (DNAfix-2014-02862).

\section{References}

[1] K. Nakatani, Y. Tor, Eds., Modified Nucleic Acids, Springer International Publishing, Cham, 2016.

[2] M. S. Gonçalves, Angewandte Chemie International Edition 2017, 56, 4655-4655.

[3] W. Xu, K. M. Chan, E. T. Kool, Nat Chem 2017, 9, 1043-1055.

[4] S. De Ornellas, J. M. Slattery, R. M. Edkins, A. Beeby, C. G. Baumann, I. J. S. Fairlamb, Organic \& Biomolecular Chemistry 2015, 13, 68-72.

[5] H. Cahová, R. Pohl, L. Bednárová, K. Nováková, J. Cvačka, M. Hocek, Organic \& Biomolecular Chemistry 2008, 6, 3657-4.

[6] L. M. Wilhelmsson, Q. Rev. Biophys. 2010, 43, 159-183.

[7] A. Dumas, G. Mata, N. W. Luedtke, in Fluorescent Analogues of Biomolecular Building Blocks: Design and Applications, John Wiley \& Sons, Ltd, Hoboken, NJ, USA, 2016, pp. 242-275.

[8] B. Y. Michel, D. Dziuba, R. Benhida, A. P. Demchenko, A. Burger, Front. Chem. 2020, 8, 14757-23.

[9] N. P. F. Barthes, I. A. Karpenko, D. Dziuba, M. Spadafora, J. Auffret, A. P. Demchenko, Y. Mély, R. Benhida, B. Y. Michel, A. Burger, RSC Adv. 2015, 5, 33536-33545. 
[10] N. P. F. Barthes, K. Gavvala, D. Dziuba, D. Bonhomme, I. A. Karpenko, A. S. DabertGay, D. Debayle, A. P. Demchenko, R. Benhida, B. Y. Michel, et al., Journal of Materials Chemistry C 2016, 4, 3010-3017.

[11] H.-N. Le, C. Zilio, G. Barnoin, N. P. F. Barthes, J.-M. Guigonis, N. Martinet, B. Y. Michel, A. Burger, Dyes and Pigments 2019, 170, 107553.

[12] D. R. Weinberg, C. J. Gagliardi, J. F. Hull, C. F. Murphy, C. A. Kent, B. C. Westlake, A. Paul, D. H. Ess, D. G. McCafferty, T. J. Meyer, Chem. Rev. 2012, 112, 4016-4093.

[13] J. R. Lakowicz, Principles of Fluorescence Spectroscopy, Springer Science \& Business Media, 2013.

[14] C. A. M. Seidel, A. Schulz, M. H. M. Sauer, The Journal of Physical Chemistry 1996, 100, 5541-5553.

[15] F. S. A. X. Peng, S. S. Pujari, Bioconjug. Chem. 2010, 21, 1629-1641.

[16] T. Aso, K. Saito, A. Suzuki, Y. Saito, Organic \& Biomolecular Chemistry 2015, 13, 10540-10547.

[17] D. Dziuba, I. A. Karpenko, N. P. F. Barthes, B. Y. Michel, A. S. Klymchenko, R. Benhida, A. P. Demchenko, Y. Mély, A. Burger, Chemistry 2014, 20, 1998-2009.

[18] F. S. A. X. Peng, M. Zulauf, Synthesis 1996, 1996, 726-730.

[19] M. Torimura, S. Kurata, K. Yamada, T. Yokomaku, Y. Kamagata, T. Kanagawa, R. Kurane, Anal Sci 2001, 17, 155-160.

[20] D. Rehm, A. Weller, Israel Journal of Chemistry 1970, 8, 259-271.

[21] B. Calitree, D. J. Donnelly, J. J. Holt, M. K. Gannon, C. L. Nygren, D. K. Sukumaran, J. Autschbach, M. R. Detty, Organometallics 2007, 26, 6248-6257.

[22] Y. Koide, Y. Urano, K. Hanaoka, T. Terai, T. Nagano, ACS Chem. Biol. 2011, 6, 600608.

[23] R. A. Marcus, The Journal of Chemical Physics 1956, 24, 966-978.

[24] R. A. Marcus, Canadian Journal of Chemistry 1959, 37, 155-163.

[25] N. J. Turro, V. Ramamurthy, J. C. Scaiano, Principles of Molecular Photochemistry: an Introduction, University Science Books, 2009.

[26] C. Reichardt, Chem. Rev. 1994, 94, 2319-2358.

[27] J. Catalán, J Phys Chem B 2009, 113, 5951-5960.

[28] S. M. Ormson, R. G. Brown, F. Vollmer, W. Rettig, "Journal of Photochemistry \& Photobiology, A: Chemistry” 1994, 81, 65-72.

[29] V. V. Shynkar, A. S. Klymchenko, E. Piémont, A. P. Demchenko, Y. Mély, The Journal of Physical Chemistry A 2004, 108, 8151-8159.

[30] A. Sougnabé, D. Lissouck, F. Fontaine-Vive, M. Nsangou, Y. Mély, A. Burger, C. A. Kenfack, RSC Advances 2020, 10, 7349-7359.

[31] My Hang V Huynh and and Thomas J Meyer, 2007, 1-61.

[32] B. Ma, P. I. Djurovich, M. E. Thompson, Coordination Chemistry Reviews 2005, 249, 1501-1510.

[33] P.-O. Gendron, F. Avaltroni, K. J. Wilkinson, J Fluoresc 2008, 18, 1093-1101.

[34] T. Dertinger, V. Pacheco, I. von der Hocht, R. Hartmann, I. Gregor, J. Enderlein, Chemphyschem 2007, 8, 433-443.

[35] I. R. Gould, R. H. Young, R. E. Moody, S. Farid, The Journal of Physical Chemistry 1991, 95, 2068-2080.

[36] I. R. Gould, R. H. Young, L. J. Mueller, S. Farid, J. Am. Chem. Soc. 1994, 116, 81768187. 
[37] I. R. Gould, D. Noukakis, L. Gomez-Jahn, R. H. Young, J. L. Goodman, S. Farid, Chemical Physics 1993, 176, 439-456.

[38] K. K. Mentel, R. M. D. Nunes, C. Serpa, L. G. Arnaut, J Phys Chem B 2015, 119, 75717578.

[39] J. Eriksen, C. S. Foote, The Journal of Physical Chemistry 1978, 82, 2659-2662.

[40] A. R. Melnikov, E. V. Kalneus, V. V. Korolev, I. G. Dranov, A. I. Kruppa, D. V. Stass, Photochem. Photobiol. Sci. 2014, 13, 1169-1179.

[41] J. Qi, D. Liu, X. Liu, S. Guan, F. Shi, H. Chang, H. He, G. Yang, Anal. Chem. 2015, 87, 5897-5904.

[42] S. Chatterjee, W. Pathmasiri, O. Plashkevych, D. Honcharenko, O. P. Varghese, M. Maiti, J. Chattopadhyaya, Organic \& Biomolecular Chemistry 2006, 4, 1675-1686.

[43] A. I. Skilitsi, D. Agathangelou, I. Shulov, J. Conyard, S. Haacke, Y. Mély, A. Klymchenko, J. Léonard, Phys Chem Chem Phys 2018, 20, 7885-7895.

[44] M. L. Horng, J. A. Gardecki, A. Papazyan, M. Maroncelli, The Journal of Physical Chemistry 1995, 99, 17311-17337.

[45] E. F. Pettersen, T. D. Goddard, C. C. Huang, G. S. Couch, D. M. Greenblatt, E. C. Meng, T. E. Ferrin, J Comput Chem 2004, 25, 1605-1612.

[46] D. Escudero, Acc. Chem. Res. 2016, 49, 1816-1824.

[47] A. C. Benniston, A. Harriman, Chemical Society Reviews 2006, 35, 169-179.

[48] G. L. Closs, J. R. Miller, Science 1988, 240, 440-447.

[49] F. Seela, H. Xiong, P. Leonard, S. Budow, Organic \& Biomolecular Chemistry 2009, 7, 1374-1387.

[50] P. Trojanowski, J. Plötner, C. Grünewald, F. F. Graupner, C. Slavov, A. J. Reuss, M. Braun, J. W. Engels, J. Wachtveitl, Physical Chemistry Chemical Physics 2014, 16, 13875-13888.

[51] Y. Saito, R. H. E. Hudson, "Journal of Photochemistry \& Photobiology, C:

Photochemistry Reviews” 2018, 36, 48-73.

[52] P. Leonard, D. Kondhare, X. Jentgens, C. Daniliuc, F. Seela, J. Org. Chem. 2019, 84, 13313-13328. 